\title{
Antioxidant effect of Beta-D-glucan-polysaccharide fractionate of Auricularia polytricha on Hyperglycaemia-Induced Kidney Dysfunction in Experimental Diabetic Nephropathy
}

\author{
Agbor C A ${ }^{1, *}$ and Anyanwu G E ${ }^{2}$ \\ ${ }^{1}$ Department of Anatomy, Faculty of Basic Medical Sciences, University of Calabar, Nigeria; ${ }^{2}$ Department of Anatomy, Faculty of Basic \\ Medical Sciences, University of Nigeria, Nigeria.
}

Received: May18, 2020; Revised: Dec 2, 2020; Accepted: January 4, 2021

\begin{abstract}
$\beta$-D-Glucan polysaccharide is a major bioactive component fractionated from Auricularia polytricha (mushroom), an edible fungus used for both nutritional and therapeutic purposes. Local Nigerian men use A. polytricha to manage diabetes related complications without accepted experimentation to ascertain the role and mechanism of action of it fractionates. This study was intended to find out the effect of $\beta$-D-Glucan polysaccharide supplementation of hyperglycaemia-induces kidney dysfunction in diabetic Wistar rat. Experiment animals were divided into four groups and received treatment as follows; Group A (treated with normal saline and served as normal control), Group B, (diabetic control animals were treated with $65 \mathrm{mg} / \mathrm{kg}$.bw of streptozotocin (STZ)), Groups C and D, (placed on $120 \mathrm{mg} / \mathrm{kg} . b \mathrm{bw}$ and $200 \mathrm{mg} / \mathrm{kg} . b w$ of $\beta$-D-Glucan polysaccharide respectively after inducing diabetics). At termination, 24 hours urine was collected and analyzed for creatinine clearance and proteinuria, both kidneys were harvested and weighed, serum was collected by cardiac puncture and analysed for serum creatinine and oxidative stress markers (Superoxide Dismutase. Catalase and Malondialdehyde), while histopathological examination was done using routine Hematoxyline and Eosine technique with the help of light microscope. The result showed that hyperglycaemic ambience caused a significant increase in serum level of oxidative stress markers along with concomitant cytoarchitectural alterations of kidney tissue and a change in values of renal parameters (serum creatinine, proteinuria, and creatinine clearance) indicating kidney dysfunction in diabetic control animals. However, decrease in activities of oxidative stress markers, reversal of alterations in kidney microstructure, and the overall improvement in values of renal parameters were observed following administration of $\beta$-D-Glucan polysaccharide in a dose-dependent manner. The present study suggests that $\beta$-D-Glucan polysaccharide supplementation can ameliorate hyperglycaemia-induced nephrotoxicity in diabetic wistar rat model.
\end{abstract}

Keywords: $\beta$-D-Glucan polysaccharide, diabetic nephropathy, Auricularia polytricha, kidney, oxidative stress

\section{Introduction}

Diabetic nephropathy is one of the major complications associated with diabetes mellitus. It is characterized by accumulation of extracellular matrix in the glomerular and tubulointerstitial compartments of the kidney which may result in renal failure (Nangaku et al. 2004). Researchers have described cellular events during diabetic nephropathy to include generation of Reactive Oxygen Specie (ROS), channeling of glucose intermediates into metabolic pathways resulting in generation of advanced glycation product and the increase in expression of transforming growth factor. The result is hyperglycaemia-induced injury from excessive generation of ROS thereby causing an alteration in intraglomerular hemodynamics (Singh et al. 2008).

Both glomerular and tubulointerstitial injuries are involved in pathogenetic mechanisms of diabetic nephropathy. Diabetic kidney injury begins with hyperglycaemia which in turn creates a redox environment in the vasculature, nephron and surrounding interstitium causing dysfunction of virtually all types of kidney cells (Phillips et al. 2003). Tubulointerstitial injury develops after glomerular injury and correlates with alteration in renal parameters such as decreased Glomerular Filtration Rate (GFR), increase in proteinuria and decrease in creatinine clearance (Nangaku et al. 2004).

Oxidative stress caused by increased levels of ROS serve as an inducer and amplifier of signaling cellular events that occur in high glucose ambience (Ha and Lee, 2005; Brownlee, 1995). ROS is required in small amounts necessary to maintain cellular homeostasis, but sustained hyperglycaemia in diabetic conditions will cause a dramatic rise in ROS thereby damaging target organs (Lee et al. 2003). Mechanism of reactive oxygen specie generation involves, high oxygen concentration reduced to water, while part of it is transformed to $\mathrm{O}_{2}{ }^{-}$in the mitochondria (Brand, 2010). This is capable of causing cellular dysfunction, apoptosis and mutations (Nishikawa et al., 2000)

There has been a shift to herbal therapy with an increasing acceptance in recent times, even among the elite confirming that that herbal therapy is potent in

\footnotetext{
*Corresponding author e-mail: agbor_cyril@yahoo.com.
} 
management ant treatment of several diseases (Anthony et al., 2006). Numerous animal and plant products have been reported to have medicinal properties (Bhatia et al., 2010). $\beta$-D-Glucan polysaccharide is a major bioactive constituent of Auricularia polytricha (mushroom), an edible fungus used globally for culinary and therapeutic purposes. $\beta$-glucans are glucose polymers polysaccharides linked together by a 1-3 linear $\beta$-glycosidic chain core and are found as a major constituent in the cell wall of fungus. They differ from each other by their length and branching structures (Stone and Clarke, 1992) and do not always exist in single form but as conjugate with other biomolecules such as amino acid, protein, lipid and nucleic acid residue. More so, its low molecular weight, chelating effect and chemical modification by biochemical intermediates makes them a strong source of exogenous antioxidant (Nie et al., 2011).

Local Nigerian men have used $A$. polytricha to manage complications in diabetic men without reliable experimentation to back up their claim. This study tried to find out the antioxidant effect of $\beta$-D-Glucan polysaccharide fractionate of $A$. polytricha on experimental diabetes nephropathy using Wistar rat model.

\section{Materials And Methods}

\subsection{Collection of Mushroom}

A. Polytricha was purchased from Etomi central market in Etung Local Government Area of Cross River State. It was then taken to Department of Biological Sciences, University of Nigeria for identification. The mushroom was dried under room temperature, powdered and then extracted with ethanol (Anyanwu and Agbor, 2020).

\subsection{Fractionation of $\beta$-D-glucan polysaccharide}

$\beta$-D-glucan polysaccharide was experimentally separated from $A$. polytricha using acetyl trimethyl ammonium bromide to form a precipitated complex with the acidic polysaccharide. It was further purified and structurally described through the use of a combination of fractional precipitation with acetic acid using ion-exchange chromatography. (Zhang et al. 2007)

\subsection{Experimental Animals}

Sixteen (16) adult male Wistar rats were weighed and grouped into four in clean cages with four rats in each. The rats were kept for two weeks of acclimatization in animal house, Department of Anatomy, Faculty of Basic Medical Sciences, University of Nigeria, Enugu Campus and allowed free access to available chow and water.

\subsection{Experimental Design}

The experimental grouping, treatment and dosage are shown in Table 1
Table 1. Experimental animals were divided into four (4) groups with 4 animals in each. Treatment received is as follows:

\begin{tabular}{llll}
\hline Group & $\begin{array}{l}\text { Number of } \\
\text { Animals }\end{array}$ & Treatment & Dose \\
\hline A & 4 & Distilled water & $3 \mathrm{mls}$ \\
\hline B & 4 & Streptozotocin $(\mathrm{STZ})$ & $65 \mathrm{mg} / \mathrm{kg} . \mathrm{bw}$ \\
\hline C & 4 & $\begin{array}{l}\text { STZ }+\beta \text {-D-glucan } \\
\text { polysaccharide }\end{array}$ & $120 \mathrm{mg} / \mathrm{kg} . \mathrm{bw}$ \\
\hline D & 4 & $\begin{array}{l}\text { STZ }+\beta \text {-D-glucan } \\
\text { polysaccharide }\end{array}$ & $200 \mathrm{mg} / \mathrm{kg} \cdot \mathrm{bw}$ \\
\hline
\end{tabular}

\subsection{Induction of Hyperglycaemia}

Diabetes was induced by administration of $65 \mathrm{mg} / \mathrm{kgbw}$ of Streptozotocin (STZ) intraperitoneally after allowing the animals to fast for 12 hours, STZ was reconstituted in $0.5 \mathrm{M}$ of Sodium citrate before administration (Ugochukwu and Babady 2003).

\subsection{Confirmation of Hyperglycaemia}

Accu-Check glucometer (Roche diagnostic, Germany) was used to confirm diabetes 3 days after STZ administration, using blood samples from the tails of experimental animals. Blood glucose levels was checked before and after induction to confirm diabetic state (Anyanwu and Agbor, 2020).

\subsection{Administration of Extract}

After two weeks of sustained hyperglycaemia, $\beta$-Dglucan polysaccharide was administered via oral gastric intubation. to experimental groups ( $\mathrm{B}, \mathrm{C}$ and $\mathrm{D})$ once daily. The experiment lasted for 2 weeks,

\subsection{Collection of Tissues and Samples for Analysis}

At termination, kidneys were harvested weighed together using an electronic weighing balance (Mettler Instrument AG, Switzerland) and suspended in buffered neutral formaldehyde for further processes with conventional histological techniques. Paraffin sections were cut at 5.0 microns using a Manual Rotary Microtome. Heamatoxylin and Eosin ( $\mathrm{H} \& \mathrm{E})$ stained sections were observed under a light microscope.

\subsection{Evaluation of Oxidative Stress Markers}

Oxidative stress markers were analyzed using blood obtained by cardiac puncture. Samples were transported to the laboratory for biochemical study. Oxidative stress marker kits (Sigma-Aldrich Products, Germany) was used to demonstrate for Superoxide Dismutase (SOD), Catalase and Malondialdehyde (MDA) (Anyanwu and Agbor, 2020).

\subsection{Analysis for Renal Parameters}

Urine was obtained from metabolic cages housing the experimental animals on the last day, before sacrifice. Creatinine auto-analyzer (Beckman Instrument Fullerton CA) was used to evaluate urine and serum creatinine levels while spectrophotometric assay was adopted in estimation of total protein (Sharma et al., 2001). 


\subsection{Statistical Analysis}

Data obtained from this study was recorded and analyzed using one way analysis of variance (ANOVA) with SPSS program (version 20). Post-hoc test was conducted using Fischer's Least Significant Difference (LSD) to determine statistical significance among groups. Probability level of $P<0.05$ was considered significant.

\section{Results}

\subsection{Weight of Kidney}

As shown in Figure 1, a significantly $(\mathrm{p}<0.05)$ lower kidney weight $(0.80 \pm 0.34 \mathrm{~g})$ was observed in diabetic control (Group B) when compared to normal control $(1.82 \pm 0.67 \mathrm{~g})$. However, hyperglycaemic animals that received $120 \mathrm{mg} / \mathrm{kg}$.bw and $200 \mathrm{mg} / \mathrm{kg}$.bw of $\beta$-D-glucan polysaccharide in Groups $\mathrm{C}$ and $\mathrm{D}$ respectively recorded kidney weights that were significantly $(\mathrm{p}<0.05)$ higher when compared to the diabetic control.
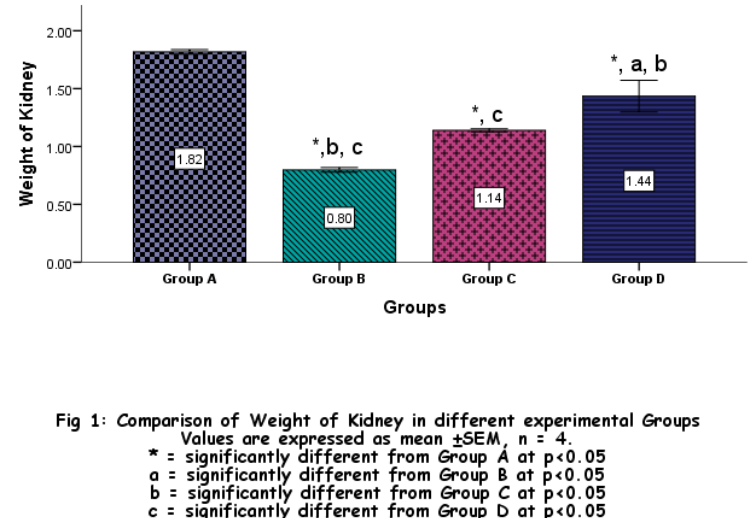

\subsection{Biochemical Analysis}

High serum SOD, Catalase and Malondialdehyde (MDA) level were recorded by the diabetic control animals and these values were significantly $(\mathrm{p}<0.05)$ higher when compared to normal control group. However, all the diabetic animals treated with graded doses of $\beta$-D-glucan polysaccharide had significantly lower SOD, catalase and Melondialdehyde activities when compared with diabetic control (Figures 2, 3 and 4).
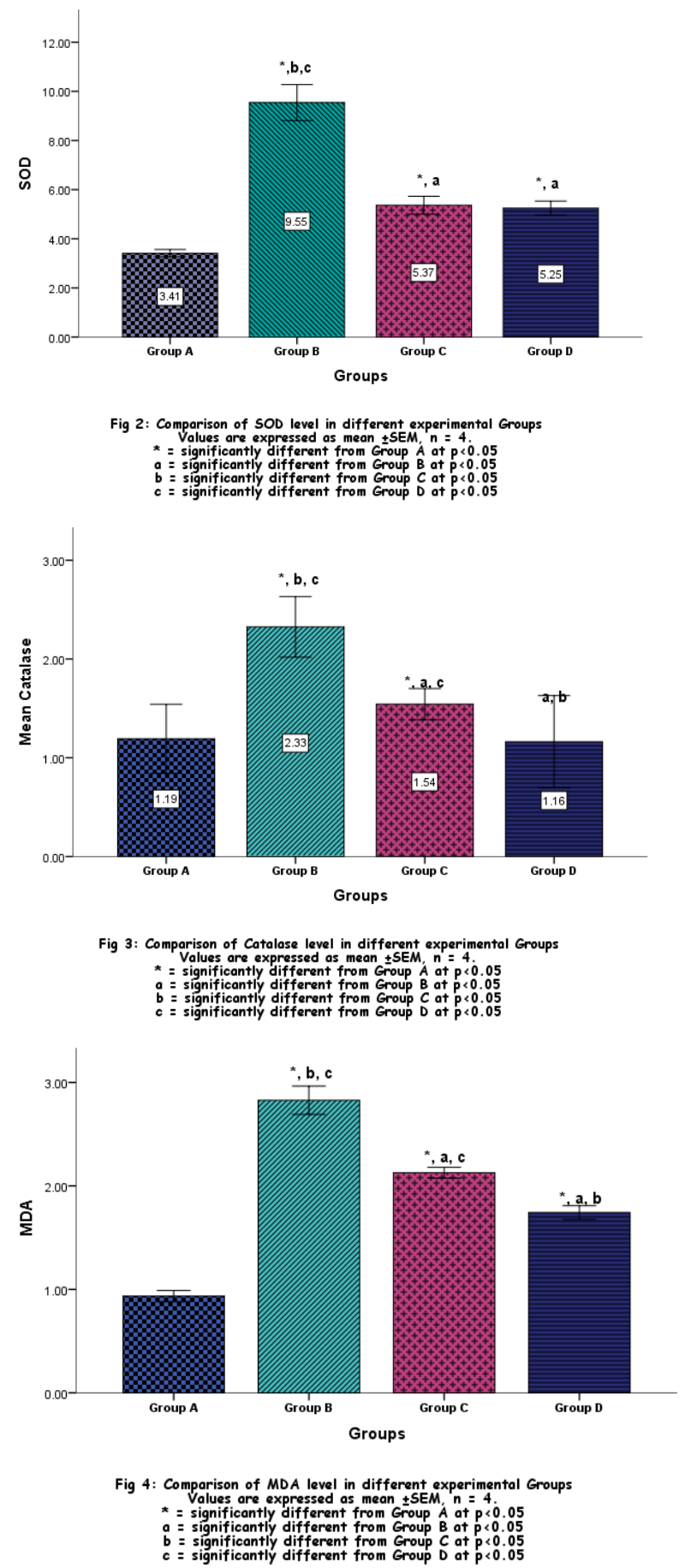


\subsection{Histopathological Examination}

Fig. 4: Histopathological Examination of Kidney - X400
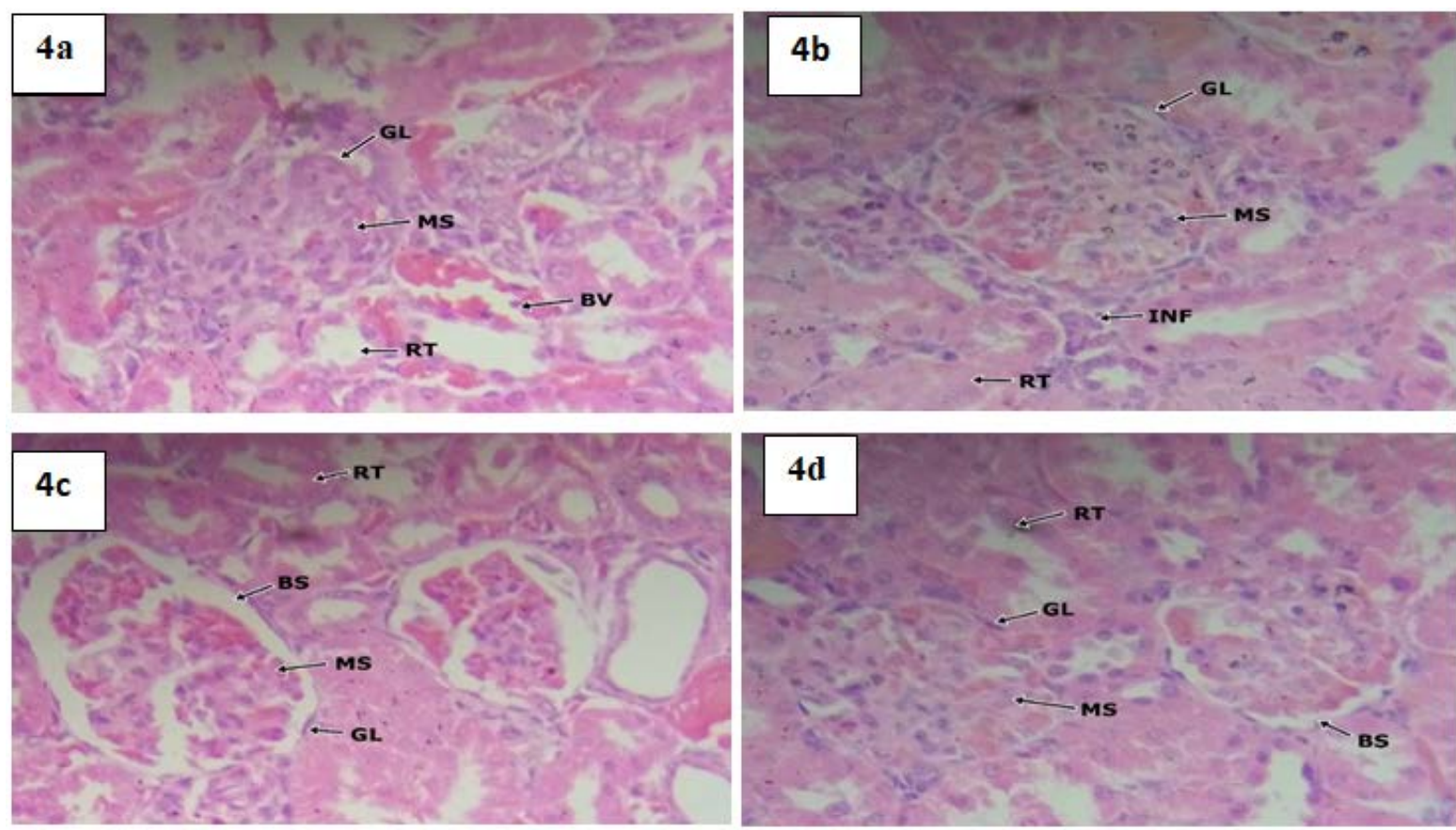

Key GL - Glomerulus MS - Mesangium RT - Renal tubuleBS - Bowman.s Space BV - Blood Vessel.

Figure 4. a) Section of the kidney in group A (Normal control) showing prominent renal tubules and glomeruli. The intervening stroma is scanty and consists of sparse interstitial cells and dilated blood vessels. The glomeruli and Bowman's space were seen intact. b) Section of the kidney of animals in Group B (Diabetic control) showing prominent renal tubules and glomeruli with diffused expansion and proliferation of mesangium, thickening of glomerular basement membrane and renal tubular hypertrophy. The intervening stroma consists of inflammatory infiltrates. The glomeruli are mildly swollen with a distorted Bowman's space. Extensive haemorrhage is seen within the mesangial matrix. c) Section of the kidney in group C (Diabetic animals placed on $120 \mathrm{mg} / \mathrm{kg}$.bw $\beta$-D-glucan polysaccharide) shows prominent renal tubules and glomeruli. The intervening stroma is scanty and consists of sparse interstitial cells and mild inflammatory infiltrates. The glomeruli are atrophic with an intact bowman space and a cellular mesangium. d) Section of the kidney in group D (Diabetic animals placed on $200 \mathrm{mg} / \mathrm{kg}$.bw $\beta$-D-glucan polysaccharide) shows prominent renal tubules and glomeruli. The intervening stroma is scanty and consists of sparse interstitial cells and mild inflammatory infiltrates. The glomeruli have intact Bowman's space and a cellular mesangium.

\subsection{Renal Parameters}

As shown in figures 6, 7 and 8, serum creatinine level $(2.58 \pm 0.44 \mathrm{mg} / \mathrm{dl})$ in diabetic control (Group B) was remarkably increased when compared to normal control $(0.69+0.84)$, proteinuria in diabetic control group was also significantly higher when compared to normal control, whereas creatinine clearance in diabetic control group was significantly $(p<0.05)$ lower when compared to normal control.

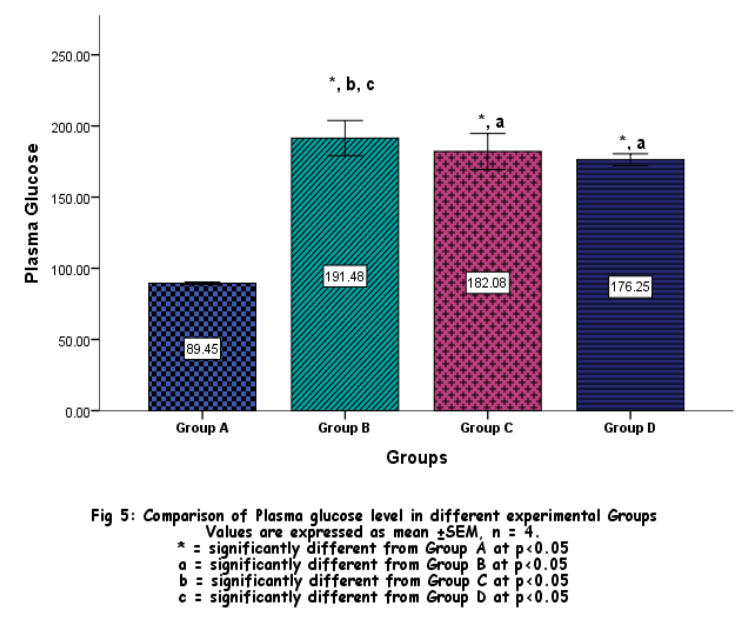



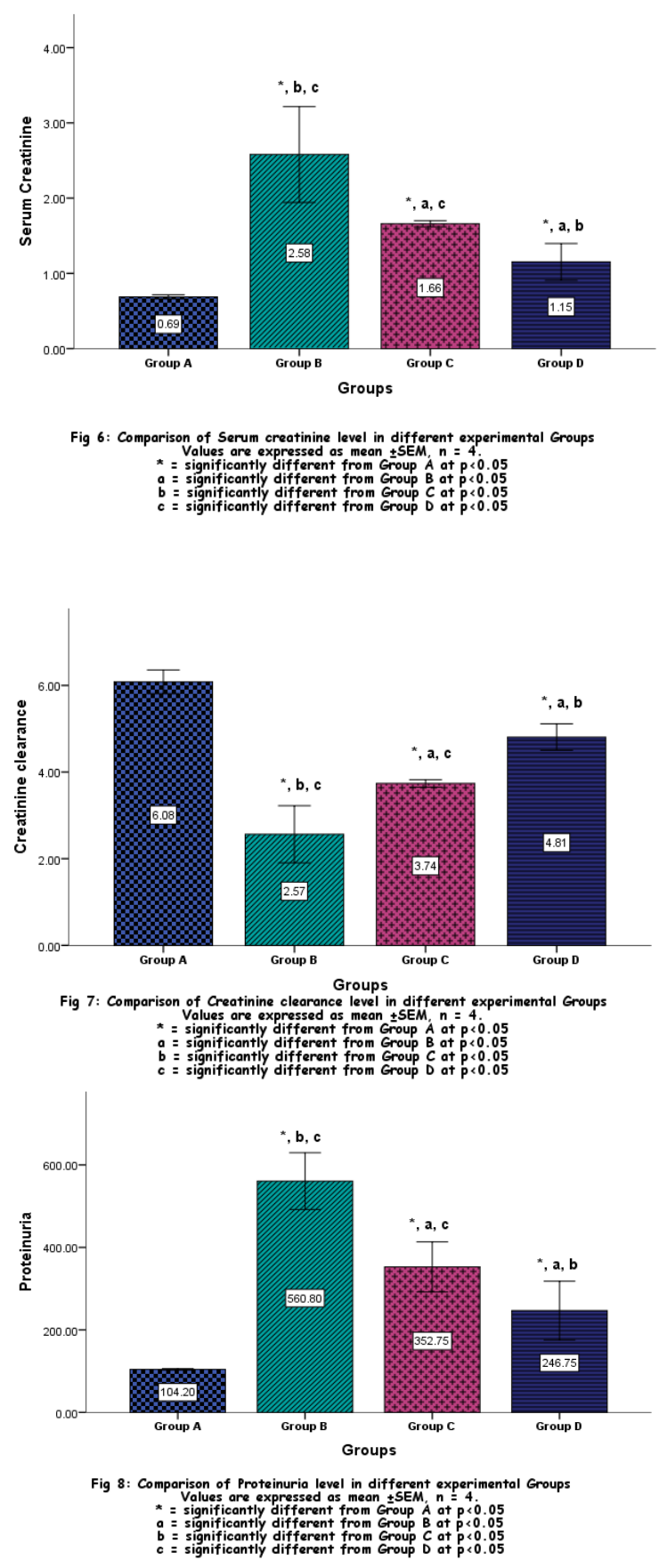

\section{Discussion}

This study has revealed that sustained hyperglycaemia within the tissue of the kidney can cause significant reduction in weight of organ. It has been demonstrated that weight of an organ depends, to a large extent, on the mass of various cells. According to Wolf (2004), hyperglycaemic injury affects all cell types in the kidney including mesangial, vascular endothelia interstitial and fibroblast cells at varying degrees. When compared to the diabetic control group, ten weeks of administration of $\beta$-Dglucan polysaccharide to diabetic rat may have caused the increase in kidney weight in a dose-dependent manner. Chang et al. (2011) have reported that A. polytricha (unfractionated fungi) is capable of inhibiting lipid peroxidation in body organs thereby restoring their weight.
There are also confirmatory reports by Sherma et al. (2001).

The remarkably higher levels of serum SOD, Catalase and melondialdehyde in group B when compared to group A confirm high hyperglycaemic ambience within the renal tissue. It has been documented that $\beta$-D-glucan polysaccharide is a strong exogenous source of antioxidant that do not exist as a single form but forms conjugates with other vital bio-molecular elements like amino acid, protein, lipid and nucleic acid residue (Nie et al., 2011). The underlying mechanism could be that $\beta$-D-glucan polysaccharide has low molecular weight and more hydroxyl group terminals capable of accepting and eliminating free radicals and these are the most important structural feature that determines the antioxidant capacity of polysaccharides. Liu et al. (2010) reported that peptide moiety of polysaccharide has been found to have high free radical scavenging activity which is a possible reason for low levels of oxidative stress markers in diabetic animals treated with $\beta$-D-glucan polysaccharide.

As shown in figure 4, Group A presented a kidney section having prominent renal tubules with an intact glomeruli (figure 4a). Histological section of kidney in diabetic control (figure 4a) group reveals diffused expansion and proliferation of mesangium, thickening of glomerular basement membrane, renal tubular hypertrophy, scanty and sparse interstitial cells with inflammatory infiltrates, distorted Bowman's space and extensive hemorrhage within the mesangial matrix as a result of hyperglycaemic injury which affects all cell types in the kidney. Fioretto and Mauer (2007) reported that glomerular, tubule-interstitial and vascular changes constitute pathogenicity of diabetic nephropathy. In this study, glomerular changes are shown in mesangial cell proliferation and thickening of glomerular basement membrane, changes in the tubulointerstitial compartment included inflammatory infiltrate and tubular hypertrophy while the changes in vascular compartment were notices in the various degrees of hemorrhage within the mesangial matrix. Hyperglycaemia-induced injury results from excessive generation of ROS causing changes in intraglomerular hemodynamics.

Extensive experimental evidence (Aitken and Krausz 2001; Lenzi et al., 1993;) have reported modification of nucleotide bases thereby causing alteration in DNA structure accompanied by deletion, cross linking and chromosomal rearrangement with its attendant consequences that ends in cellular damage.

Findings from this study also show that hyperglycaemia-induced alterations which are signs of kidney damage were minimized and reversed in a dose dependent manner following administration of $\beta$-D-Glucan polysaccharide as shown in figures $4 \mathrm{c}$ and $4 \mathrm{~d}$. Even though a comprehensive investigation into the mechanism of action of $\beta$-D-Glucan polysaccharide is still lacking, its low molecular weight and existence in conjugate forms may trigger an interaction with certain receptors that may result in some specific therapeutic signaling pathway to benefit host organ. $\beta$-D-Glucan polysaccharide have also been shown to possess lipid peroxidation inhibitory effect which make it capable of preventing damage of cell membrane. Renard et al (2001) reported similar findings.

Renal parameters obtained from the diabetic control group are indicators of renal dysfunction. Results obtained 
from renal parameters in different experimental groups show a correlation between these parameters and hyperglycaemia-induced kidney injury. However, these values were reversed following $\beta$-D-Glucan polysaccharide administration. Improvement in these renal parameters following administration of $\beta$-D-glucan polysaccharide may have reflected the reversal in hyperglyaemia-induced distortion of glomerular, tubolointerstitial and vascular compartments in the kidney tissue.

In conclusion, the findings from this research revealed that $\beta$-D-Glucan polysaccharide fractionate of $A$. polytricha was capable of ameliorating hyperglycaemiainduced kidney damage in experimental diabetic nephropathy models. This research therefore suggest that $\beta$-D-Glucan polysaccharide may reduce the risk of kidney dysfunction in diabetic men.

\section{Acknowledgment}

I acknowledge the technical support from the Department of Histopathology, University of Calabar Teaching Hospital, Nigeria and Endocrinology Laboratory in the Department of Biochemistry, University of Calabar Nigeria.

\section{Funding}

This research had no external source of funding.

\section{Competing Interest}

All authors in this research are not by any means linked to any funding body, and so there was a complete absence of competing interest.

\section{Authors' Contributions}

All authors gave equal contribution to this research.

\section{Ethical Clearance}

Ethical clearance for this research was obtained from the Ethical Committee, Faculty of Basic Medical Sciences, University of Nigeria, Enugu Campus, Nigeria.

\section{References}

Aitken RJ and Krause C. 2001. Oxidative stress, DNA damage and the Y- chromosome. Reproduction 122: 497-506.

Anthony BO, Oladipo AL, Adedoyin KL and Tajudin IA. 2006. Phytochemistry and spermatogenic potential of aqueous extracts of Cissus Populnear. The Sci World J, 6:2140-2146.

Anyanwu GE and Agbor CA. 2020. Assessment of testicular histomorphometric parameters and reticular fibre density on testicular tissue of diabetic Wister rat placed on Auricularia Polytricha. Jordan J. Biol. Sci. 13(Supplementary): 709-714

Bhatia DK, Sharma AK, Pathania PC and Khauduri NC. 2010. Antifertility effect of crude extract of Adiantum Lunulatum on Reproductve-organs of male wister rats. Int. J Biol Forum, 2 (2):88-93.
Brand MD. 2010. The sites and topology of mitochondrial superoxide production. Exper Gerontol, vol 45, No 7-8, pp. 466472.

Brownlee M. 1995. Advanced protein glycosylation in diabetes and aging. Annu. Rev. Med.; 46:223-234.

Chang JS, Kim HJ, Bae JT, Park SH, Kim SE and Kim OM. 2011. Inhibition effects of Auricularia auricula-judae methanol extract on lipid peroxidation and liver damage in benzo(a)pyrene-treated mice. J Korean Soc Food Sci Nut 27: 712-717.

Fioretto P, Mauer M. 2007. Histopathology of diabetic nephropathy. Semin. Nephrol. 27:195-207.

Ha H, Lee HB. 2005. Reactive oxygen species amplify glucose signaling in renal cells cultured under high glucose and in diabetic nephropathy. Nephrol. 10:S7-S10.

Lee HB, Yu MI-RA, Yang Y, Jiang Z, Ha H. 2003. Reactive oxygen species-regulated signaling pathways in diabetic nephropathy. J. Am. Soc. Nephrol. 14:S241-S245.

Lenzi A, Cualosso F, Gandini L, Lombardo F and Dondero F. 1993. Placebo controlled, double-blind, cross-over trial of glutathione therapy in male infertility. Human Rep 9: 2044-2050.

Liu W, Wang H, Pang X, Yao W and Gao X. 2010. Characterization and antioxidant activity of two low-molecularweight polysaccharides purified from the fruiting bodies of Ganoderma lucidum . Int J of Biol Macromol. 46(4):451-457. doi: 10.1016/j.ijbiomac.2010.02.006.

Nangaku M. 2004. Mechanisms of tubulointerstitial injury in the kidney: final common pathways to end stage renal failure. Int Med. 43:9-17.

Nie S.-P., Xie M.-Y., 2011. A review on the isolation and structure of tea polysaccharides and their bioactivities. Food Hydrocolloids. 25(2):144-149.

doi: 10.1016/j.foodhyd.2010.04.010.

Nishikawa T, Edelstein D and Brownlee M. 2000. The missing link: a single unifying mechanism for diabetes complications. The Kidney J, 77, pp.526-530.

Phillips AO. 2003. The role of renal proximal tubular cells in diabetic nephropathy. Curr Diabetes Rep. 3:491-496.

Renard CM. Baron A, Guyot S and Drilleau JF. 2001. Interactions between apple cell walls and native apple polyphenols: quantification and some consequences. Int J Biol Macromol. 29(2):115-125. doi: 10.1016/s0141-8130(01)00155-6

Sharma RK, Pasqualotto AE and Nelson DR. 2001. Relationship between seminal white blood cell count and oxidative stress in men treated at an infertility clinic. J Androl, 22:575-583.

Singh DK, Winocour P, Farrington K. 2008. Mechanisms of disease: the hypoxic tubular hypothesis of diabetic nephropathy. Nat. Clin. Pract. Nephrol. 4:216-226.

Stone BA, Clarke AE. 1992. Chemistry and biology of (1,3)-Dglucans. Victoria, Australia.: La Trobe University Press.

Ugochukwu NH and Babady NE. 2003. Antihyperglycaemic effect of aqueous and ethanolic extracts of Gongronema latifolium leaves on glucose and glycogen metabolism in livers of normal and streptozotocin induced diabetic rats. Life sci, 73 (150): 19251938.

Wolf G. 2004. New insights into the pathophysiology of diabetic nephropathy: from hemodynamics to molecular pathology. Eur. J. Clin. Investig. 34:785-796.

Zhang H, Wang ZY, Yang L, Yang X, Wang X and Zhang Z. 2007. In vitro antioxidant activities of sulfated derivatives of polysaccharides extracted from Auricularia auricular. Int $\mathrm{J}$ of $\mathrm{Mol}$ Sci 12: 3288-3302. 